\title{
The Potential of Cloud Computing as an Alternative Technology for SMEs in South Africa
}

\author{
Mpho J. Mohlameane and Nkqubela L. Ruxwana
}

\begin{abstract}
SMEs operate in competitive markets for business growth and sustainability. Information and Communication Technology (ICT) solutions have the potential to increase SMEs competitiveness thus contributes towards business sustainability. However SMEs mainly use the traditional ICT solutions to stay competitive. This leads to several challenges that they face in implementing ICT solutions, such as lack of capital, skilled staff, and so forth. The eminent challenge is that ICTs are expensive to procure and maintain. Considering these challenges, there is a need for cost-effective alternative ICT solutions that when implemented, can improve SMEs competitiveness. This paper investigates SMEs challenges regarding the adoption and use of traditional (on-premise) ICT solutions, and the potential of cloud computing as an alternative technology. The study adopted qualitative research approach, where case study research designs was used consisting of three SMEs. The data was collected through interviews, expert reviews, literature and questionnaires, where a total of 19 participants were used. The findings reveals that cloud computing happens to be a cost-effective alternative solution compared to traditional (on-premise) ICT solutions.
\end{abstract}

Index Terms-Cloud computing, competitiveness, ICT, SMEs.

\section{INTRODUCTION}

ICT is an essential component in the "knowledge economy" [1], which is the critical factor for any business success and sustained competitive advantage. Hence, it is critical for SMEs to become a part of this economy in order to compete and thrive in the future.

The notion of cloud computing becomes even more relevant for businesses operating in developing countries, especially SMEs which are expected to compete with larger cooperates to gain sustainable advantage. The relevancy of cloud computing in SMEs is therefore that it promises to have potentials for cost saving and improved efficiency [2].

This paper starts by defining SMEs within the study context. The notion of cloud computing is discussed, focusing on cloud computing definition and brief discussion of cloud deployment models and services. The methodology section discusses the approach used to collect and analyse data. The results section present and discusses current implemented ICT solutions within SMEs, challenges regarding the adoption and use of ICT, as well as cloud

Manuscript received March 5, 2013, revised May 15, 2013. This work was supported in part by the Department of Informatics at Tshwane University of Technology.

M. J. Mohlameane is with P-Cubed, Bryanston 2194 South Africa (e-mail: mpho.mohlameane@gmail.com).

N. L. Ruxwana is with Vodacom, Midrand 1687 South Africa (e-mail: nkqubz@yahoo.co.uk). computing as an alternative ICT solutions for SMEs.

\section{SMES DEFINITION}

The National Small Business Act defines SMEs by making use of three parameters, namely: number of employees, annual turnover and gross assets (excluding fixed assets) [3]. The National Small Business Act defines small enterprise with the number of employees amounting to fewer than 50 and medium enterprises with the number of employee amounting to fewer than 100 to 200 [3].

For the purpose of this study, the abbreviation SME was employed as the study focused on small and medium- sized enterprises, which are registered with the South African Receiver of Revenue and have the potential to adopt ICT services. The study excluded very small and micro enterprises, which have fewer than 10 employees and do not have any potential of adopting ICT services.

\section{The Notion OF Cloud COMPUTING}

\section{A. Definition}

Different authors, organisations and technologist have tried to define cloud computing in various ways. In a nutshell, cloud computing is a new computing model whereby computing services such as data storage, software applications, and so forth, are accessed over the Internet. The National Institute of Standard and Technology (NIST) provided us with the formal definition of cloud computing, and that is:

"Cloud computing is a model for enabling convenient, on-demand network access to a shared pool of configurable computing resources (e.g., networks, servers, storage, applications, and services) that can be rapidly provisioned and released with minimal management effort or service-provider interaction" [4].

\section{B. Cloud Computing Deployment Models}

Cloud deployment models are classified into four types: public, private, hybrid and community. Public cloud consists of resources that are shared among cloud subscribers and the shared resources are accessible over the Internet [5]. Examples of public cloud are Google Apps, Amazon Web Services (AWS), Salesforce, etc. Private clouds are largely designed and deployed within the enterprise to be used by internal users only [6]. Hybrid cloud consists of elements of public and private cloud [7], [8]. NIST define hybrid cloud as "a composition of two or more distinct cloud infrastructures (private, community, or public) that remain unique entities, 
but are bound together by standardized or proprietary technology that enables data and application portability (e.g., cloud bursting for load balancing between clouds)" [4]. Community cloud is formed by organisations and institutions that typical shares the same set of values, such as shared mission and goals, security requirements, policies and compliance [5], [7], [9].

\section{Cloud Computing Services Model}

Cloud services models can be classified into three types: Platform as a Service (PaaS), Infrastructure as a Service (IaaS), and Software as a Service (SaaS) [10]. Platform as a Service cloud delivery model provides infrastructure and platform in the cloud for application developers to build deploy and run applications on the Internet and users are billed per-use [11]. Examples of PaaS include Microsoft Windows Azure and Google App Engine. Infrastructure as a Service is the delivery of hardware infrastructure (i.e., servers, storage, and so forth.) as a service, accessible over the internet and hosted by the cloud provider [12]. Examples of IaaS offerings include (EC2) and Secure Storage Service (S3). Software as a Service is the delivery of software such as ERP, Customer Relationship Management (CRM), E-mail and Collaboration Software, among others, over the Internet as a service [5], [13]. Examples of SaaS offerings include Microsoft Office 365, Google Apps, Hosted Exchange Server, etc. which are offered on a subscription-based pricing model, thus paying for only what you use.

\section{RESEARCH METHODOLOGY}

\section{A. Research Philosophy}

This study followed phenomenological philosophical approach in order to gain an in depth understanding of the complexity of phenomena investigated in a natural setting and real-life context. Phenomenology is concerned with the study of individual experience regarding particular phenomena [14].

\section{B. Research Approach}

For the purpose of this study qualitative research was adopted and served to help the researcher understand the phenomena under study in a natural setting.

In this study, a multiple-case study design was employed. Three different SMEs around Gauteng were part of the unit of analysis. The three enterprises are from different industries- insurance, financial and engineering. Because the operation of companies and their adoption levels for innovations differ per industry, it was necessary to choose companies from different business industries in South Africa in order to obtain rich qualitative data.

\section{Sampling Design and Research Participants}

A sample is a representative part of the targeted population that is methodologically selected to participate in a study [15]. Purposive sampling was applied when sampling the case studies and various participants. According to Babbie [16], purposive sampling involves the selection of the units to be observed on the basis of your own judgment about which ones will be the most useful or representative.
Three SMEs were purposefully selected as units of analysis and there were five participants per SME business, thus total number of participants from all SMEs was fifteen. The primary data was sourced through questionnaires and interviews. To improve findings validity, four experts in the field were asked to participate and they were selected based on availability and access.

\section{Data Collection Methods}

McNiff [17] states that there are various sources of data however, the data collection method should be appropriate for a specific research study. The use of multiple data collection methods increases the credibility of data [18], [19]. In order to increase the credibility and reliability of data, the researcher used multiple data collection methods - namely: interviews, expert reviews, questionnaires and literature findings.

\section{E. Data Analysis Methods}

In this study, the process of data analysis was based on data reduction and interpretation. Creswell [20] asserts that data analysis in qualitative research involves data preparation, coding and interpreting data. The study analysis followed three phases where triangulation was adopted to answer the study objectives. Within these phases the case study analysis templates, namely within-case, cross-case and holistic-case, as recommended by Creswell [20] were adopted.

\section{FINDINGS AND DisCUSSIONS}

\section{A. Current ICT Solutions Implemented in SMEs Sector}

In the questionnaire, respondents were asked to identify ICT solutions implemented in their business. As depicted in Fig. 1, all (100\%) respondents indicated that their companies have implemented ICT and mostly use computers, e-mail service and Microsoft office suite; $93 \%$ of respondents indicated to mostly use both the Internet and network infrastructure; $80 \%$ indicated to be using the mainframe system and servers; while only 33\% are mostly using enterprise resource planning and CRM applications. Figure 1 below illustrates these findings:

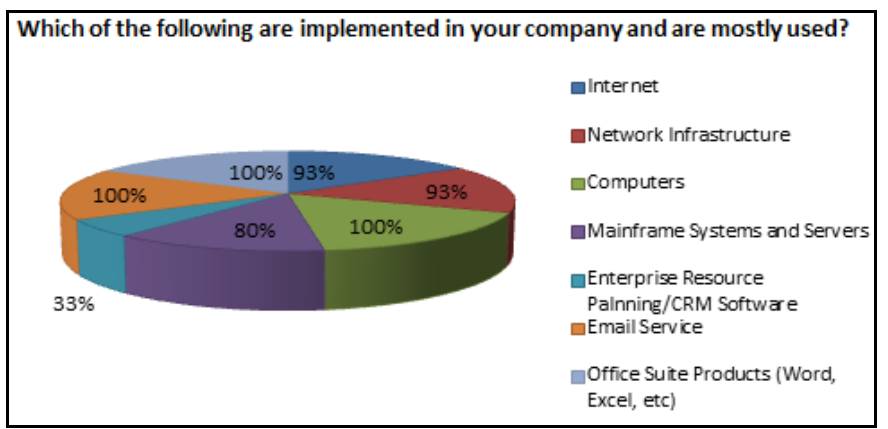

Fig. 1. ICT Solutions implemented in the company.

It is evident from Fig. 1 above that there is a widespread adoption of ICT solutions among SMEs. The participants stated that their companies have implemented and use some of the ICT solutions such as e-mail services, computers, office suite, servers and network infrastructure. The Internet revealed to be mostly used in the SME sector. The findings 
also indicate that there is low adoption rate of software solutions such as ERP and CRM applications. This can be due to anecdotal evidence that these software solutions might not be compatible with the current implemented ICT systems; the cost of their adoption, as well as the lacking need to have such solutions as they have limited number of clients.

There were similarities in the interview findings of the different SMEs regarding the current implemented and mostly used ICT solutions, where solutions used included Internet, Microsoft office, network infrastructure, e-mail services, computers, data warehouse and business intelligence software: "We have network infrastructure, email services, antivirus solutions, office suite products and servers. All these services play a critical role within the company. Email is very critical because it is strongly used for communication purposes".

Literature findings revealed that there is a wide range of software applications and systems that are used within the SME sector such as CRM, Operations Management, Vendor Relationships, Strategy Implementation, Network Management, Enterprise Resource Planning (ERP), and so forth [21]-[23]. The study conducted by Ismail et al. [21] reveals that SMEs have implemented and are using ICT solutions such as Internet, email services, accounting packages, human resource packages, and enterprise resource planning and customer relationship management.

The overall findings reveal that the Internet, computers, servers, network infrastructure, office suite products, CRM applications and e-mail services are the most commonly used ICT solutions within SMEs. The availability of ICT within SMEs enables them to efficiently develop product and services that are of highly quality and which can enable them to improve their competitive advantage

\section{B. Challenges Regarding the Adoption and Use of ICT Solutions}

There are several challenges that hinder the adoption and use of ICTs, these include factors such as insufficient capital, a lack of ICT skills, performance issues, downtime and disaster recovery, and the cost of ICT solutions. When asked in the interview about the challenges regarding the adoption of ICT, SMEs had to say: "The challenges we have with the implementation of ICT solutions is Disaster recovery (DR), Piloting and Replication and this negatively affect our SLA'S with clients. When implementing new ICT solutions, we do not put measures in place for disaster recovery for in case the implementation of new solution fails".

Literature review revealed some of the challenges regarding the adoption and use of ICT. One of the key barriers of ICT adoption is a lack of ICT benefits awareness and ICT skills [24], [25]. Modimogale et al. [26] and Mpye et al. [27] assert that SMEs perceive ICT as an expensive tool to implement and maintain. The cost of ICT is, therefore considered to be a barrier for adoption.

The experts have acknowledged that there are several challenges regarding the adoption and use of ICT solutions, the cost of ICT solutions and a lack of ICT skills to support ICT solutions being key concerns: "The most common challenge is the expensive cost of ICT services. The current ICT model is still based on capital expenditure and SMEs are required to make some upfront capital investment to implement and use ICT services".

There is an inevitable consensus amongst case study findings, experts and literature reviews that there are challenges regarding the implementation and use of ICTs among SMEs. The findings revealed a direct link on the common challenges that have a greater hindrance and effect on SMEs adoption of ICT, including the lack of sufficient capital, lack of ICT skills and high costs of ICT solutions. The higher costs and complexities of ICTs make it even more difficult for SMEs to implement and effectively use ICT solutions

\section{Cloud Computing as an Alternative ICT Solutions}

The study findings reveal that cloud computing promise to be a cost-effective alternative ICT solution compared to traditional (on-premise) ICT solution. By traditional (on-premise) ICT solutions, we refer to ICT solutions that are hosted and managed internally by SMEs.

The experts acknowledged the cost-effectiveness of cloud computing for SMEs, and strongly recommended the adoption of cloud computing services. When asked about the benefits of cloud computing, experts were quick to emphasise the cost-effectiveness of cloud computing as a key benefit, and the following comments were made:

Respondent 1: "Cloud computing is one of the best ideas ever available for SMEs because it is seems to be more affordable compared to IT services that we normally use. This is because services are provided on a subscription basis and this this gives SMEs flexibility to manage cost".

Respondent 2: "Cloud services are more affordable compared to normal ICT services and this is because they are hosted by the provider. This on its own can save SMEs licensing costs, support and administration cost as well as server and hardware costs".

Respondent 3: "The key benefit of cloud computing for SMEs is cost savings. Most SMEs perceive the normal ICT services to be expensive to adopt and maintain. Cloud computing now allows them to adopt cloud services which are more affordable".

Respondent 4: "The idea behind cloud computing is to shift focus from capital expenditure to operational expenditure. This is a perfect opportunity for SMEs who are experiencing issues regarding adopting and using normal ICT services due to high costs and technical issues".

Experts assert that because cloud services are hosted by a provider, this can save software licensing, labour and maintenance costs. This will also shift the security and risk management. The cost of infrastructure, capacity; redundancy and disaster recovery costs will also move to the service provider, offering considerable costs savings for the SMEs. Moreover, experts mentioned that cloud computing initiate a shift from Capital Expenditure (CAPEX) to Operational Expenditure (OPEX), giving SMEs the agility to manage their cost effectively.

There is a widely accepted view, especially in the SMEs sector, that ICTs are expensive to setup, operate and manage [26], [28]. The cost of ICT has been viewed as a barrier for adoption among SMEs [27]. However findings reveal that cloud computing promise to be cost-effective alternative 
solution that can enable SMEs to invest in ICT. For instance, Osterman Research [29] conducted a study and compared hosted exchange server and on-premise exchange server and found out that hosted exchange server happens to cost less compared to on-premises servers. Furthermore Osterman Research revealed that within a period of three years, an SME with 100 users can save more than half of the cost of on-premises ICT expenditure and this is a significant saving on costs.

In concurrence with Osterman Research [29], Schadler [30] compared on-premises and cloud-based e-mail services prices and found that Google Apps appears to be less costly compared to on-premises e-mail services. It costs around $\$ 5$ per user per month and $\$ 50$ per user per year. What makes cloud-based e-mail service cheaper than those on-premises is that, on-premises e-mail requires upfront initial investment, support and maintenance.

The findings of this study reveal that SMEs mostly use desktop office suite products such as Microsoft Excel, Word and PowerPoint which are also offered on the cloud. For instance Google offers cloud-based office applications such as Google Apps at a cost of $\$ 5$ per user per month. With Google Apps, SMEs can get access to messaging applications such as Gmail, Google Calendar and Google Groups for business. SMEs can also get collaboration applications such as Google Docs, Sites and Video for business. On the other side, Microsoft Office 2010 prices ranges from $\$ 120$ to $\$ 680$ depending on the product version (i.e., professional, home and business). This example shows a considerable cost savings for those in use of cloud computing than the traditional approaches of ICTs.

Of those SMEs interviewed, all of them mostly use computers. The problem with computers is that their value often depreciates too quickly [31]. As they depreciate, they no longer have that computing power to process a number of tasks at a quick rate than they used to. Furthermore computers require upfront and maintenance costs. However, companies like Vodacom offer cloud-based solutions such as "Office in the Cloud" on a subscription-based cost model, with monthly premium staring from R600 per user per month [32].

Vodacom's Office in the Cloud consists of "a thin client terminal, flat screen monitor, keyboard and mouse, Cisco VoIP enabled phone handset, an HP Picture 6 or HP LaserJet M1536 MFP Mono Printer, access to the applications portal comprising of a wide choice of business application" [32]. This product from Vodacom consists of cloud services (IaaS and SaaS) that SMEs might require to successfully operate their businesses. What it is more interesting about this product is that, SMEs can considerably save on software, infrastructure and hardware costs.

\section{CONCLUSIONS}

ICT solutions can play a strategic role towards the improvement of SMEs competitiveness. However SMEs are reluctant to invest in ICT solutions due to lack of ICT skills, high cost of ICT solutions, performance issues, downtime and disaster recovery and so forth. Of those SMEs which have ICT in their internal environment, they are faced with several challenges such as lack of sufficient ICT and problem solving skills to provide satisfactory ICT support, and lack of sufficient capital to procure bespoke ICT solutions. The consequence of these issues negatively affect business operation as in most cases they have no alternative option or failover at the time of ICT failures.

Of the recent ICT evolutions, cloud computing happens to be an alternative ICT solutions compared to traditional (on-premise) ICTs. The traditional cost model of on-premise ICT is characterised by software licensing cost, support and maintenance cost as well as upfront capital investment.

Cloud computing can enable SMEs to invest in bespoke ICT solutions that can help contribute towards SMEs competitiveness. Cloud computing is considered to be cost-effective as there is no cost associated with software licensing, upfront ICT investment costs, hardware and infrastructure cost. It is therefore concluded that cloud computing serves as an alternative ICT solution compared to traditional (on-premise) ICT solutions.

\section{RECOMMENDATIONS}

It is recommended that SMEs should start considering cloud computing solution as opposed to traditional (on-premise) ICT solutions. However SMEs will need to increase their knowledge and understanding of cloud computing which will empower them to know the pros and cons of cloud computing, as well as to evaluate whether it is a suitable ICT solutions based on their business model.

SMEs can improve their knowledge and understanding of cloud computing through several communication channels, such as mass media and interpersonal. Mass media includes newspapers, internet, television or radio. Interpersonal communication channel can consist of communication between two or more people. SMEs should make use of cloud service providers to present workshops/demonstration on cloud computing concepts and benefits. This will help address some of the challenges and concerns that SMEs have regarding cloud computing.

In addition, cloud computing service providers can play a critical role towards increasing the awareness of cloud computing, by developing cloud computing proof of concepts for SMEs as well as conducting cloud computing presentation to SMEs. These presentations can be beneficial to SMEs; firstly, they introduce the concepts of cloud computing, and secondly, they present cloud computing benefits, and thirdly, they provide demonstration on how to use cloud computing services. Furthermore, SMEs can increase their awareness of cloud computing through learning from other SMEs who have already implemented cloud computing services, their experiences, benefits and challenges with the adoption of cloud computing.

Cloud computing services can be put on trial as most providers such as Microsoft, Salesforce, Google, and so forth, offer limited trial periods for SMEs to experiment cloud computing services. Google offers a 30-day free trial version for their Google Apps for SaaS solutions. Salesforce offers a 30-day free trial period for their CRM SaaS solutions. Microsoft also offers a 30-days free trial version for Office 365 SaaS solutions. Therefore as part of increasing the 
awareness and knowledge of cloud computing, it is recommended that SMEs start to learn to make use of such cloud computing applications as they will also have an opportunity to understand the benefits that cloud computing can bring to their business.

\section{REFERENCES}

[1] B. Ritchie and C. Brindle, "ICT adoption by SMEs: implications for relationships and management," New Technology, Work and Employment, vol. 20, no. 3, pp. 205-217, 2005.

[2] M. Armbrust, A. Fox, R. Griffith, A. Joseph, R, Katz, A. Konwinski, G. Lee, D. Patterson, A. Rabkin, I. Stoica, and M. Zaharia, Above the clouds: A Berkeley view of cloud computing, University of California, Berkeley, Technical Report, 2009.

[3] H. Falkena, I. Abedian, and M. Blottnitz, "SMEs' access to finance in South Africa," The Task Group of the Policy Board for Financial Services and Regulation, 2002.

[4] National Institute of Standards and Technology. (March 2012), The NIST Definition of cloud computing. [Online]. Available: http://csrc.nist.gov/publications/nistpubs/800-145/SP800-145.pdf.

[5] M. Carroll, A. Van der Merwe, and P. Kotze, "Secure cloud computing: Benefits, risks and controls," in Proc. the 2011 Information Security for South Africa (ISSA 2011) Conference, pp. 1-9. August 15-17, 2011.

[6] S. Ramgovind, M. Ellof, and E.Smith, "The management of security in Cloud computing," in Proc. Information Security for South Asia (ISSA), 2010, pp.1-7.

[7] G. Conway and E. Curry, "Managing cloud computing: a life cycle approach," in Proc. 2nd International Conference on Cloud Computing and Services Science, Porto, 2012.

[8] S. Chowhan and R. Saxena, "Customer relationship management from the business strategy perspective with the application of cloud computing," in Proc. DYNAA, vol. 2, no. 1, 2011.

[9] K. Junck and M. Rahman, "Cloud computing avoids downfall of application service providers," International Journal of Information Technology Convergence and Services, vol. 1, pp. 1-20, 2011.

[10] S. Qureshi and M. Kamal, "Role of cloud computing interventions for micro-enterprise growth: implications for global development," in Proce. the Fourth Annual SIG GlobDev Workshop: Fourth Annual SIG GlobDev Workshop, Shanghai, December 3, 2011.

[11] M. Boniface, B. Nasser, J. Papay, S. Phillips, A. Servin, Z. Zlatev, K. Yang, G. Katsaros, K. Konstanteli, G. Kousiouris, A. Menychtas, D. Kyriazis, and S. Gogouvitis, "Platform-as-a-service architecture for real-time quality of service management in clouds," in Proc. Fifth International Conference on Internet and Web Applications and Services, pp. 155-160, May 2010.

[12] S. Bhardwaj, L. Jain, and S. Jain, "Cloud computing: A study of infrastructure as a service (IAAS)," International Journal of engineering and information Technology, vol. 2, pp. 60-63, 2010.

[13] W. Dai, "The Impact of Emerging Technologies on Small and. Medium Enterprises (SMEs)," Journal of Business Systems, Governance and Ethics, vol. 4, pp. 54-60, 2009.

[14] S. Lester (May 2011). An introduction to phenomenological research [Online]. Available: http://www.sld.demon.co.uk/resmethy.pdf.

[15] D. R. Cooper and P. S. Schindler. Business Research Methods, 8th ed. New York: McGraw Hill, 2003

[16] E. Babbie. The Practice of Social Research. Belmont, California: Wadsworth, 2005.

[17] J. McNiff. (October 2011). Action research for professional development.

[18] M. Patton, Qualitative Evaluation and Research Methods, 3rd ed. Thousand Oaks, CA: Sage Publications, Inc., 2001.

[19] R. K. Yin, Case study research method, 3rd ed. Thousand Oaks, CA: Sage, 2003.

[20] J. W. Creswell, Social Research. London: Sage Publications, 2007.

[21] R. Ismail, R. Jeffery, and J. Van Belle, "Using ICT as a value adding tool in south african SMEs," Journal of African Research in Business \& Technology, vol. 2011, pp. 1-11, 2011.
[22] K. Johnston, S. Kabanda, S. Adams, and E. Davids, "How SMEs in Western Cape of South Africa Use ICT," Management of Engineering \& Technology, pp.1043-1051, 2008.

[23] R. Ashrafi and M. Murtaza, "Use and Impact of ICT on SMEs in Oman," The Electronic Journal Information Systems Evaluation, vol 11, no. 3, pp. $125-138,2008$.

[24] S. Mutula and P. Van Brakel, "ICT Skills readiness for the emerging global digital economy among small businesses in developing countries: case study of Botswana," Library Hi Tech, vol. 24, pp. 231-245, 2007.

[25] M. Kapurubandara and R. Lawson. (March 2012). Barriers Adopting ICT and E-commerce with SMEs in Developing Countries: An Exploratory Study in Sri Lanka. [Online]. Available: http://www.esmaeilkhou.com/articles/9-SriLanka-2006.pdf.

[26] L. Modimogale and J. Kroeze, "The role of ict within small and medium enterprises in Gauteng," Communication of IBIMA Journal, vol. 2011, pp.1-13, 2011

[27] D. Mpye, S. Osman and J. Van Belle. Barriers to ICT Adoption for Micro-businesses in South Africa. Eight Annual Global Information Technology Management Association (GITMA) World Conference, Napoli, Italy, June 17-19, 2007

[28] K. Mpofu, D. Milne, and L. Watkins-Mathys, "ICT adoption and development of e-business among SMEs in South Africa," United Kingdom: Buckinghamshire New University, 2009.

[29] Osterman Research. (March 2012). The Case for Hosted Exchange [Online]. Available: http://www.ostermanresearch.com/whitepapers/or_or1109b.pdf.

[30] T. Schadler. (February 2012). Should Your Email Live In The Cloud? A Comparative Cost Analysis. [Online]. Available: http://static.googleusercontent.com/external_content/untrusted_dlcp/ www.google.com/en//a/help/intl/en/admins/pdf/forrester_cloud_email _cost_analysis.pdf.

[31] M. Doms, W. Dunn, S. Oliner, and D. Sichel, "How fast do personal computers depreciate? Concepts and new estimates," in Proc. Tax Policy and the Economy, Washington, DC, November 4, 2003.

[32] Livdigital. (April 2012). Vodacom takes its own Office into the cloud from R600 and up monthly. [Online]. Available http://www.livdigital.co.za/independent/2011/08/04/vodacom-takes-it s-own-office-into-the-cloud.

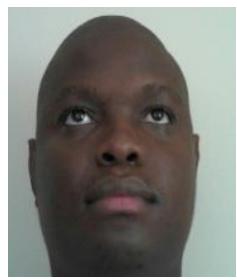

Mpho J. Mohlameane was born in Pretoria on the $19^{\text {th }}$ of September 1982. Mohlameane currently holds National Diploma in Software Development (2004), Bachelor Degree in Software Development (2010) and Master's Degree in Business Information systems (2012). All the qualifications were obtained from Tshwane University of Technology, Pretoria, South Africa. Mohlameane's major field of study is in information systems.

$\mathrm{He}$ is current employed at P-Cubed in Bryanston, South Africa as C\# developer

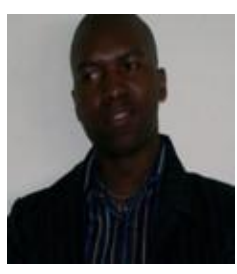

Nkqubela L. Ruxwana was born in Eastern Cape on the $25^{\text {th }}$ of December 1983. Dr. Ruxwana currently holds National Diploma in Engineering Computer Systems, Bachelor Degree in Computer Systems, and Masters in Information Systems from Tshwane University of Technology, Pretoria, South Africa. Dr. Ruxwana also holds Master's Degree in Business Leadership (University of South Africa) and a PhD in Information Technology from Nelson Mandela Metropolitan University (NMMU), South Africa.

Currently he is ICT Manager at Vodacom in South Africa and a Research Supervisor at Tshwane University of Technology, with extensive industry and research experience in the domains such as business analysis, strategy, project management, business intelligence, enterprise architecture, cloud computing, ICT4D, and health informatics. 